TITLE:

\title{
Direct actuation of cantilever in aqueous solutions by electrostatic force using high-frequency electric fields
}

\section{AUTHOR(S):}

Umeda, Ken-ichi; Kobayashi, Kei; Matsushige, Kazumi; Yamada, Hirofumi

\section{CITATION:}

Umeda, Ken-ichi ... [et al]. Direct actuation of cantilever in aqueous solutions by electrostatic force using high-frequency electric fields. Applied Physics Letters 2012, 101(12): 123112.

ISSUE DATE:

2012-09-19

URL:

http://hdl.handle.net/2433/187964

\section{RIGHT:}

(c) 2012 American Institute of Physics. This article may be downloaded for personal use only. Any other use requires prior permission of the author and the American Institute of Physics. 


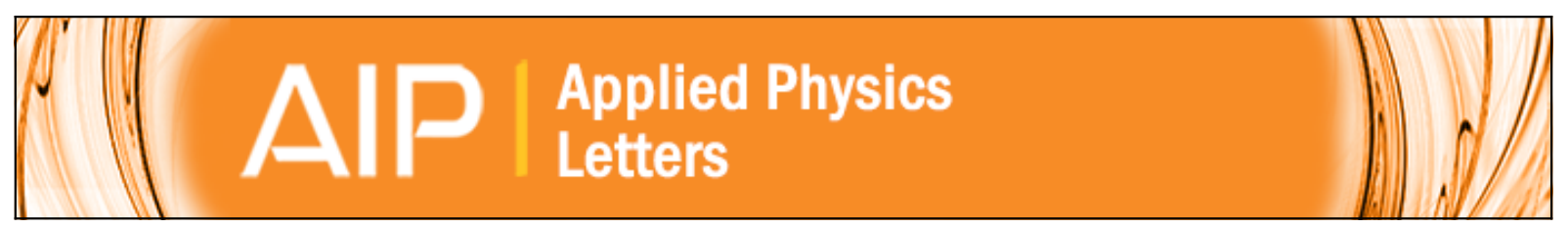

\section{Direct actuation of cantilever in aqueous solutions by electrostatic force using high- frequency electric fields}

Ken-ichi Umeda, Kei Kobayashi, Kazumi Matsushige, and Hirofumi Yamada

Citation: Applied Physics Letters 101, 123112 (2012); doi: 10.1063/1.4754289

View online: http://dx.doi.org/10.1063/1.4754289

View Table of Contents: http://scitation.aip.org/content/aip/journal/apl/101/12?ver=pdfcov

Published by the AIP Publishing

\section{Articles you may be interested in}

Fabrication and operation of an electrostatic actuator for controlling nanometer-scale gaps in collapsed cantilever heterostructures

Appl. Phys. Lett. 102, 243508 (2013); 10.1063/1.4812185

Adomian series solution for computing the effect of size dependency of material characteristics on the internal stress field of cantilever nano-actuators in the presence of intermolecular force

AIP Conf. Proc. 1479, 2411 (2012); 10.1063/1.4756681

Self-driving capacitive cantilevers for high-frequency atomic force microscopy

Appl. Phys. Lett. 100, 053110 (2012); 10.1063/1.3679684

Tip-sample distance control using photothermal actuation of a small cantilever for high-speed atomic force microscopy

Rev. Sci. Instrum. 78, 083702 (2007); 10.1063/1.2766825

Atomic force microscopy using an integrated comb-shape electrostatic actuator for high-speed feedback motion Appl. Phys. Lett. 76, 3139 (2000); 10.1063/1.126549

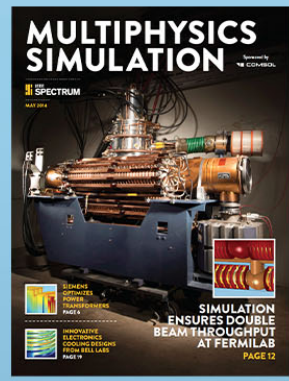




\title{
Direct actuation of cantilever in aqueous solutions by electrostatic force using high-frequency electric fields
}

\author{
Ken-ichi Umeda, ${ }^{1}$ Kei Kobayashi, ${ }^{2}$ Kazumi Matsushige, ${ }^{1}$ and Hirofumi Yamada ${ }^{1}$ \\ ${ }^{1}$ Department of Electronic Science and Engineering, Kyoto University, Katsura, Nishikyo, Kyoto 615-8510, \\ Japan \\ ${ }^{2}$ Office of Society-Academia Collaboration for Innovation, Kyoto University, Katsura, Nishikyo, \\ Kyoto 615-8520, Japan
}

(Received 12 June 2012; accepted 7 September 2012; published online 19 September 2012)

\begin{abstract}
We recently developed a method to directly actuate a cantilever in aqueous solutions by electrostatic force [K.-I. Umeda et al., Appl. Phys. Express 3, 065205 (2010)]. However, the cantilever was actuated by surface stress in a low frequency regime. We solved this problem by applying amplitudemodulated high-frequency electric fields, which actuates the cantilever solely by electrostatic force. The time variations of the self-oscillation frequency of a cantilever and the Au(111) surface images by frequency-modulation atomic force microscopy using acoustic, photothermal, and the improved electrostatic actuation methods were compared, which demonstrates the advantages of the last method such as stability and simplicity in instrumentation. (C) 2012 American Institute of Physics.

[http://dx.doi.org/10.1063/1.4754289]
\end{abstract}

Recent development of high-resolution frequency-modulation atomic force microscopy (FM-AFM) in liquid environments ${ }^{1,2}$ has enabled molecular-resolution real-space imaging of biomolecules in physiological environments. In principle, FM-AFM detects the interaction forces between a cantilever tip and sample as the resonance frequency shift of the cantilever. A piezoelectric actuator has been often used for exciting the cantilever oscillation in liquids as well as in vacuum environments and the ambient conditions. Since the mechanical $Q$-factor of the cantilever is extremely low in liquid environments due to the hydrodynamic interaction between the cantilever and the liquid, not only the resonance of the cantilever but the resonances of various mechanical parts composing a cantilever holder and/or a liquid cell are also excited at the same time. These spurious resonance peaks are often referred to as "forest of peaks." 3 The non-ideal frequency response of the cantilever causes various problems such as coupling of the conservative and the dissipative forces ${ }^{4}$ and jump in the self-oscillation frequency. ${ }^{5}$ Moreover, a phase delay at the resonance frequency causes a deviation of the self-oscillation frequency from the actual resonance frequency, which hinders quantitative force measurements. ${ }^{6}$ Several efforts have been reported on the development of cantilever holders with reduced spurious peaks; however, it is still difficult to obtain an intrinsic frequency response as a damped harmonic oscillator in liquids by indirect acoustic actuation using the piezoelectric actuators. $^{7-9}$ On the other hand, various direct actuation methods such as magnetic ${ }^{10,11}$ and photothermal ${ }^{12}$ actuation methods and those using an acoustic radiation force $^{13}$ and a Lorentz force ${ }^{14}$ have been developed, in which driving forces directly act only onto the cantilever to avoid the spurious peaks. Since most of these methods require complex instrumentation or the use of specially designed cantilevers, many researchers remain to use the acoustic actuation method for its simplicity.

We recently developed an electrostatic actuation method of a cantilever to overcome above-mentioned difficulties. ${ }^{15}$ This method is applicable for actuating metal-coated cantile- vers and simply requires an optically transparent indium-tinoxide (ITO) electrode on a glass window of the liquid cell. When an alternating bias voltage was applied between a backside metal coating of a cantilever and the ITO electrode, the cantilever was actuated by electrostatic force; however, in polar liquid media such as aqueous solutions, the cantilever was actuated by surface stress ${ }^{16}$ as well as electrostatic force depending on the frequency range. ${ }^{15}$ Typically the cantilever actuation was governed by the surface stress at a frequency range lower than tens of $\mathrm{kHz}$ in water. Because of the surface stress, the cantilever did not show an intrinsic frequency response as a damped oscillator in the low frequency range. Moreover the surface stress also causes an additional phase delay in the frequency range up to $1 \mathrm{MHz}$, which remains problematic for quantitative force measurements as mentioned above. 6,17

In this letter, we introduce a direct cantilever actuation method where the cantilever is actuated solely by electrostatic force, without affected by surface stress even in aqueous solutions. This method is basically applicable to metalcoated cantilevers, and it only requires a counter electrode, which is either a conductive sample surface or the ITO glass window. The method presented here fundamentally produces an intrinsic frequency response without any phase delay, and its actuation efficiency is prominent in a polar liquid medium, particularly in water, whose relative dielectric constant is about 80 .

The electrostatic force between the tip and sample can be described as

$$
F_{\mathrm{esf}}=\frac{1}{2} \frac{\partial C_{\mathrm{ts}}}{\partial z} V^{2}
$$

where $C_{\mathrm{ts}}$ and $z$ are the tip-sample capacitance and distance and $V$ is a bias voltage. When a voltage having two similar high-frequency (HF) components is applied between the tip and sample, the cantilever is actuated not only at the two frequency components ${ }^{15}$ but also at a beat frequency of the two 
HF components by the quadratic nature of the electrostatic force. ${ }^{18}$ In pure water, for instance, the surface stress becomes dominant in the frequency range lower than tens of $\mathrm{kHz}$ while the electrostatic force is dominant in the frequency range higher than tens of kHz. Therefore, it is expected that the cantilever can be actuated at the beat frequency solely by the electrostatic force when the two frequency components are chosen at frequencies such as those higher than $1 \mathrm{MHz}$. This method requires two HF signal generators; however it is also possible to actuate the cantilever by applying an amplitudemodulated (AM) HF voltage. In the latter method, the cantilever is actuated at a modulation frequency.

Here we consider the case when the bias voltage given by the following equation is applied:

$$
V=\left(V_{\mathrm{HF}}+\Delta V_{\mathrm{HF}} \cos \omega_{\mathrm{m}} t\right) \cos \omega_{\mathrm{HF}} t
$$

where $\omega_{\mathrm{m}}$ and $\omega_{\mathrm{HF}}$ are modulation and carrier angular frequencies, respectively. The induced electrostatic force is composed of various frequency components; however, since $\omega_{\mathrm{HF}}$ is much higher than the resonance angular frequency of a cantilever $\omega_{0}$, most of the components other than that at $\omega_{\mathrm{m}}$, which actuates the cantilever, are out of the frequency range of concern. The electrostatic force on a cantilever caused by the AM-HF voltage becomes

$$
\begin{aligned}
F_{\mathrm{esf}}^{\mathrm{AM}}= & \frac{1}{2} \frac{\partial C_{\mathrm{ts}}}{\partial z}\left[V_{\mathrm{HF}} \Delta V_{\mathrm{HF}} \cos \omega_{\mathrm{m}} t\right. \\
& \left.+\frac{\Delta V_{\mathrm{HF}}^{2}}{4}\left(\cos 2 \omega_{\mathrm{m}} t+1\right)+\frac{V_{\mathrm{HF}}^{2}}{2}\right],
\end{aligned}
$$

where we omit the components of whose frequencies are much higher than $\omega_{0}$.

Figure 1 shows a schematic of FM-AFM in liquids using the AM-HF electrostatic actuation method. We used a modified commercial AFM head (Shimadzu SPM-9600) with home-built electronics. ${ }^{19,20}$ We used a highly doped conductive silicon cantilever with a backside Au coating (Nanosensors PPP-NCHAuD). Note that we prefer the coated cantilevers for better optical sensor performance, but the method can also be applied to highly doped silicon cantilevers without metal coat-

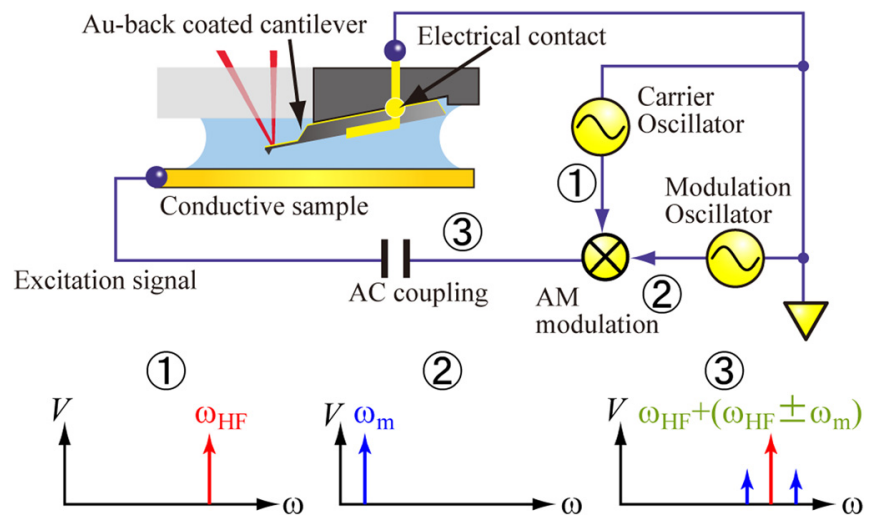

FIG. 1. Schematic of FM-AFM in liquids using AM-HF electrostatic actuation method. The AM-HF voltage was applied to a conductive sample as a counter electrode through an isolation capacitor, while a metal-coated cantilever was electrically grounded. Frequency spectra of HF carrier signal, modulation signal, and AM-HF signal are schematically shown below. ing. As a counter electrode, we used conductive samples in this study; however, it can be the ITO electrode on the cell window as mentioned above. An HF voltage signal was generated from a function synthesizer (Tektronix AFG3022B), and its amplitude was modulated with an analog multiplier (Analog Devices AD734). It should be noted that the AM-HF signal does not contain a frequency component at $\omega_{\mathrm{m}}$, as described in Eq. (2) and illustrated in Fig. 1. The signal was applied to the conducting sample through an isolation capacitor while the cantilever was electrically grounded.

Figure 2 shows the frequency response curves of a cantilever obtained by acoustic, photothermal, and the AM-HF electrostatic actuation methods. The spring constant $\left(k_{z}\right)$ of the cantilever was $29 \mathrm{~N} / \mathrm{m}$, calibrated using Sader's method, ${ }^{21}$ and the optical lever sensitivity was calibrated by fitting the thermal noise spectrum to the damped harmonic oscillator model. ${ }^{22}$ The frequency response curves were recorded by using a lock-in amplifier (AMETEK 7280). Figure 2(a) shows the frequency response curves obtained by acoustic actuation method using a piezoelectric plate attached very close to the cantilever. There are many spurious peaks in the amplitude curve, which were caused by mechanical resonances of the liquid cell including the cantilever holder. The phase varied in a complicated way as a function of the actuation frequency. Figure 2(b) shows the frequency response curves obtained by
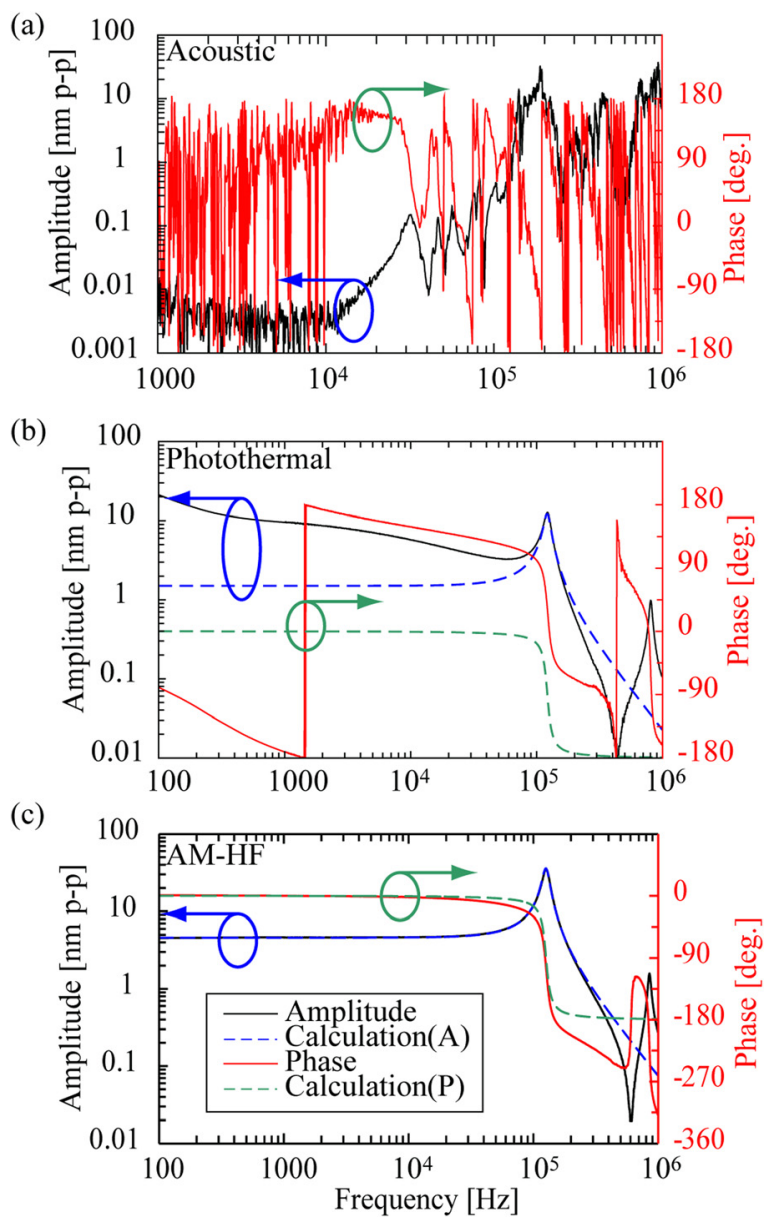

FIG. 2. Frequency response curves of the amplitude and phase of the cantilever oscillation actuated by (a) acoustic, (b) photothermal, and (c) AM-HF electrostatic actuation methods (solid curves). The curves were fitted to theoretical equation (3) (broken curves). 


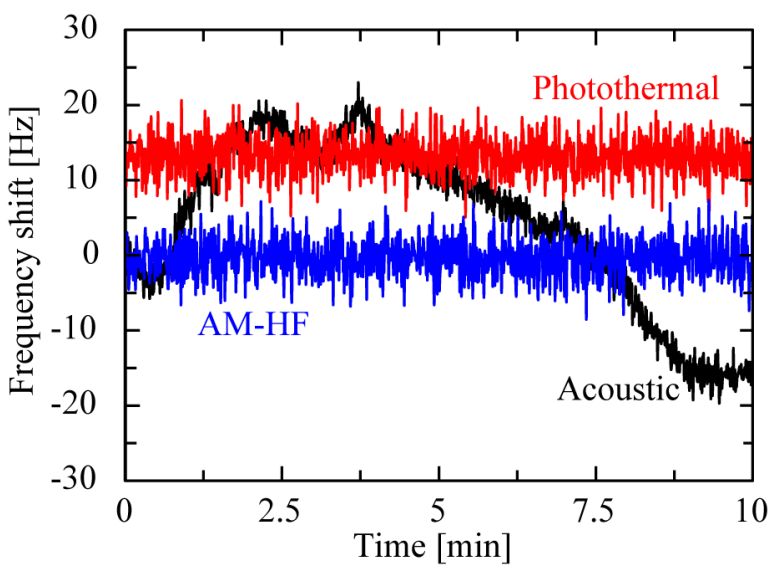

FIG. 3. Time variation in the self-oscillation frequency of a cantilever actuated by acoustic, photothermal, and AM-HF electrostatic actuation methods. The photothermal data is shown with an offset.

photothermal actuation method. The details of the photothermal actuation setup is given in Ref. 6 . The modulation amplitude of the laser power was $12 \mathrm{~mW}$ peak-to-peak with the average of $6 \mathrm{~mW}$. The amplitude curve in Fig. 2(b) shows only one resonance peak. The resonance peak was fitted to a theoretical equation using the damped oscillator model,

$$
A=G_{\text {cl }}(f) F_{\text {esf } \_\mathrm{m}}=\frac{Q}{Q\left(1-\left(f / f_{0}\right)^{2}\right)+\mathrm{i}\left(f / f_{0}\right)} \frac{F_{\text {esf } \_\mathrm{m}}}{k_{z}},
$$

where $f_{0}=\omega_{0} / 2 \pi$ and $Q$ were the resonance frequency and $Q$-factor, respectively. $F_{\text {esf_m }}$ is the electrostatic force component at $\omega_{\mathrm{m}}$, the first term in Eq. (3). The amplitude and phase curves do not match with the theoretical curves because of the finite time constant of the thermal conduction. ${ }^{23,24}$ Figure 2(c) shows the frequency response curves obtained by the AM-HF electrostatic actuation method. An AM-HF signal was applied between the cantilever and a $\mathrm{Pt}$ plate of a counter electrode located $20 \mu \mathrm{m}$ beneath the cantilever. The frequency response curves were measured as a function of the modulation frequency. The amplitude of the $\mathrm{HF}$ signal (carrier) $V_{\mathrm{RF}}$ was $6.5 \mathrm{~V}$ peak-to-zero and the modulation depth was $100 \%$ ( $\Delta V_{\mathrm{HF}}$ is equal to $\left.V_{\mathrm{HF}}\right)$. The amplitude curve again shows a single resonance peak, from which $f_{0}$ and $Q$ were determined as $126.344 \mathrm{kHz}$ and 7.8 , respectively. The effect of the surface stress in the low frequency range ${ }^{15}$ was no longer observed, and the experimental curves were almost perfectly fitted to the theoretical curves. The curves show that the intrinsic frequency response of the cantilever was obtained by the AM-HF electrostatic actuation method, except for a slight phase delay due to the limited bandwidth of the optical displacement sensor electronics, and the response of the second resonance mode.

If the carrier frequency $\left(f_{\mathrm{RF}}=\omega_{\mathrm{RF}} / 2 \pi\right)$ is lower than the characteristic dielectric relaxation frequency $\left(f_{\mathrm{c}}\right)$ that is calculated from the resistivity and dielectric constant of a solvent, $f_{\mathrm{c}}=(2 \pi \rho \varepsilon)^{-1}$, the HF signal is effectively applied to the electric double layer, and the cantilever is actuated by the surface stress at $f_{\mathrm{HF}}{ }^{15,25}$ However, by choosing $f_{\mathrm{HF}}$ greater than $f_{\mathrm{c}}$, the cantilever is solely actuated by the electrostatic force between the tip and counter electrode. In principle, the AM-HF actuation method can be applied in almost all liquid media as long as the effective carrier frequency is chosen. For example, $f_{\mathrm{c}}$ is about $3 \mathrm{MHz}$ for $1 \mathrm{~mol} / \mathrm{l} \mathrm{KCl}$ aqueous solution.

Figure 3 shows time variation in the self-oscillation frequency of the cantilever actuated by acoustic, photothermal, and AM-HF electrostatic actuation methods in pure water. The counter electrode was a Pt plate. The oscillation amplitude of the cantilever was $5 \mathrm{~nm}$ peak-to-peak. The curve obtained by the acoustic actuation showed a variation as large as tens of $\mathrm{Hz}$ during the period of the measurement because of the fluctuation of the shape of the water drop in the open liquid cell. ${ }^{17}$ On the other hand, the curves obtained (a) Acoustic
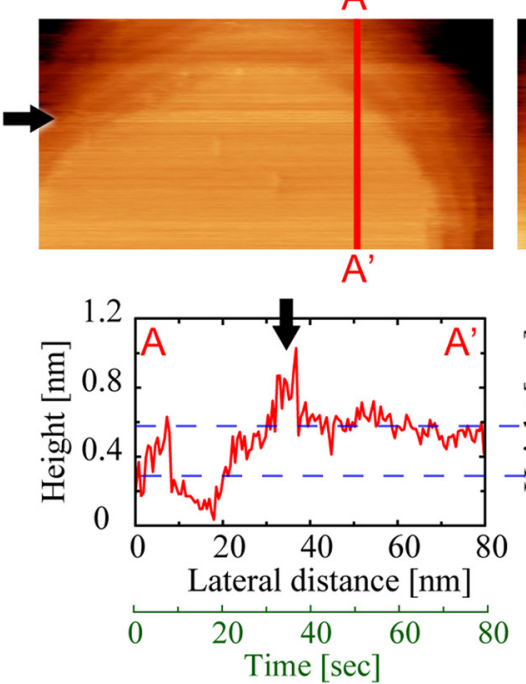

(b) Photothermal
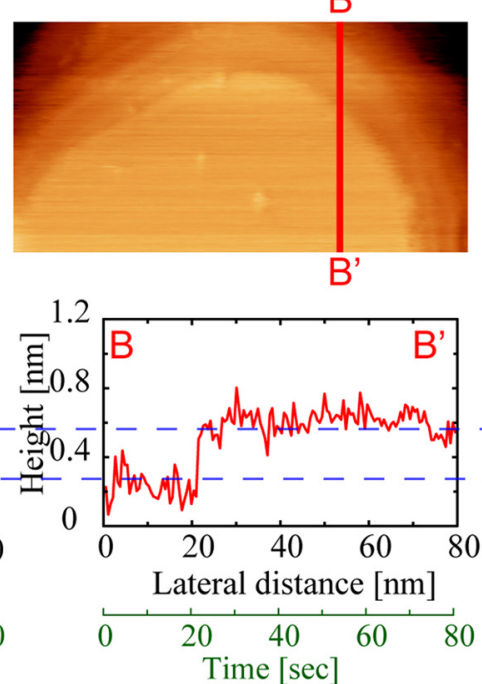

(c) AM-HF
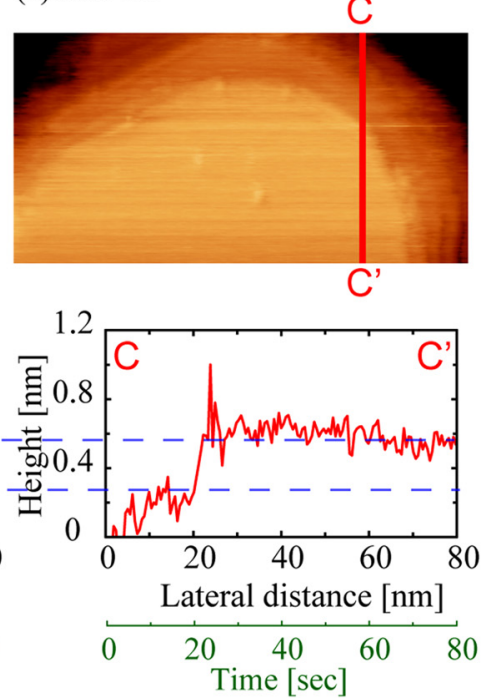

FIG. 4. FM-AFM images of the Au(111) surface in pure water obtained by (a) acoustic, (b) photothermal, and (c) AM-HF electrostatic actuation methods. The images were acquired in the constant frequency shift mode $(\Delta f=+350 \mathrm{~Hz})$ and showed without any image processing except for a tilt correction. Scanned area was $275 \mathrm{~nm} \times 139 \mathrm{~nm}$. Cross-sectional profile along a line along the slow scan direction in each image is also shown. Corresponding elapsed time calculated from the tip velocity is also labeled on the horizontal axis. The blue broken lines represent a monoatomic step height of the Au(111) surface as guides for the eyes. The black arrow indicates one of the positions at which some fluctuation in the self-oscillation frequency occurred. 
by the photothermal and AM-HF electrostatic actuation methods did not show significant variation over $10 \mathrm{~min}$, because the cantilever was self-oscillated at the intrinsic resonance frequency of the cantilever, which is required for high-resolution AFM imaging.

Finally, we performed FM-AFM imaging of the $\mathrm{Au}(111)$ surface epitaxially grown on a muscovite mica by acoustic, photothermal, and HF electrostatic actuation methods in water. The cantilever was oscillated in the constant amplitude mode at $0.8 \mathrm{~nm}$ peak-to-peak. The images were acquired in the constant frequency shift mode with a tip velocity of about 1070 nm/s. Figures 4(a)-4(c) show FM-AFM images acquired by acoustic, photothermal, and AM-HF electrostatic actuation methods, respectively. Cross-sectional profile of each line along the slow scan direction is also shown below. A monoatomic step with a height of about $0.3 \mathrm{~nm}$ was observed in each image and cross-sectional profile. Because of a nonlinear thermal drift of the tip-sample distance during the measurements, some height fluctuations were also observed in the FM-AFM images and profiles; however, the height fluctuation caused by the fluctuation of the self-oscillation frequency as shown in Fig. 3 was suppressed in the photothermal and AM-HF electrostatic actuation methods, which brought clearer monoatomic step features.

In conclusion, we developed HF electrostatic actuation method for a cantilever in liquid media which has a great advantage such as stability and simplicity over acoustic and photothermal actuation methods. The method provides an intrinsic frequency response of a cantilever, which is free from the effect of the surface stress. Since the electrostatic force is acting between a whole cantilever and a counter electrode, there is no fluctuation or jump in the selfoscillation frequency, which allows us a precise resonance frequency measurement for high-resolution FM-AFM imaging, as well as for cantilever sensor applications, ${ }^{26}$ in various liquid media.

This work was supported by Grant-in-Aids for Scientific Research from the Ministry of Education, Culture, Sports, Science and Technology of Japan, SENTAN Program of the
Japan Science and Technology Agency, and Global COE Program of the Japanese Society for the Promotion of Science.

${ }^{1}$ T. Fukuma, K. Kobayashi, K. Matsushige, and H. Yamada, Appl. Phys. Lett. 87, 034101 (2005).

${ }^{2}$ H. Yamada, K. Kobayashi, T. Fukuma, Y. Hirata, T. Kajita, and K. Matsushige, Appl. Phys. Express 2, 095007 (2009).

${ }^{3}$ T. E. Schaffer, J. P. Cleveland, F. Ohnesorge, D. A. Walters, and P. K. Hansma, J. Appl. Phys. 80, 3622 (1996).

${ }^{4}$ J. E. Sader and S. P. Jarvis, Phys. Rev. B 74, 195424 (2006).

${ }^{5}$ X. Xu and A. Raman, J. Appl. Phys. 102, 034303 (2007).

${ }^{6}$ K. Kobayashi, H. Yamada, and K. Matsushige, Rev. Sci. Instrum. 82, 033702 (2011).

${ }^{7}$ H. Asakawa and T. Fukuma, Rev. Sci. Instrum. 80, 103703 (2009).

${ }^{8}$ C. Carrasco, P. Ares, P. J. de Pablo, and J. Gomez-Herrero, Rev. Sci. Instrum. 79, 126106 (2008).

${ }^{9}$ A. Maali, C. Hurth, T. Cohen-Bouhacina, G. Couturier, and J. P. Aime, Appl. Phys. Lett. 88, 163504 (2006).

${ }^{10}$ W. H. Han, S. M. Lindsay, and T. W. Jing, Appl. Phys. Lett. 69, 4111 (1996).

${ }^{11}$ I. Revenko and R. Proksch, J. Appl. Phys. 87, 526 (2000).

${ }^{12}$ G. C. Ratcliff, D. A. Erie, and R. Superfine, Appl. Phys. Lett. 72, 1911 (1998).

${ }^{13}$ F. L. Degertekin, A. G. Onaran, M. Balantekin, W. Lee, N. A. Hall, and C. F. Quate, Appl. Phys. Lett. 87, 213109 (2005).

${ }^{14}$ A. Buguin, O. Du Roure, and P. Silberzan, Appl. Phys. Lett. 78, 2982 (2001).

${ }^{15}$ K.-I. Umeda, N. Oyabu, K. Kobayashi, Y. Hirata, K. Matsushige, and H. Yamada, Appl. Phys. Express 3, 065205 (2010).

${ }^{16}$ R. Raiteri and H.-J. Butt, J. Phys. Chem. 99, 15728 (1995).

${ }^{17}$ A. Labuda, K. Kobayashi, D. Kiracofe, K. Suzuki, P. H. Grütter, and H. Yamada, AIP Adv. 1, 022136 (2011).

${ }^{18}$ H. Yokoyama, M. J. Jeffery, and T. Inoue, Jpn. J. Appl. Phys. 32, L1845 (1993).

${ }^{19}$ T. Fukuma, M. Kimura, K. Kobayashi, K. Matsushige, and H. Yamada, Rev. Sci. Instrum. 76, 053704 (2005).

${ }^{20}$ K. Kobayashi, H. Yamada, H. Itoh, T. Horiuchi, and K. Matsushige, Rev. Sci. Instrum. 72, 4383 (2001).

${ }^{21}$ J. E. Sader, J. W. M. Chon, and P. Mulvaney, Rev. Sci. Instrum. 70, 3967 (1999).

${ }^{22}$ M. J. Higgins, R. Proksch, J. E. Sader, M. Polcik, S. Mc Endoo, J. P. Cleveland, and S. P. Jarvis, Rev. Sci. Instrum. 77, 013701 (2006).

${ }^{23}$ D. M. Todorović, B. Cretin, Y. Q. Song, and P. Vairac, J. Appl. Phys. 107, 023516 (2010).

${ }^{24}$ V. Pini, B. Tiribilli, C. M. C. Gambi, and M. Vassalli, Phys. Rev. B 81, 054302 (2010).

${ }^{25}$ T. L. Sounart, T. A. Michalske, and K. R. Zavadil, J. Microelectromech. Syst. 14, 125 (2005).

${ }^{26}$ N. V. Lavrik, M. J. Sepaniak, and P. G. Datskos, Rev. Sci. Instrum. 75, 2229 (2004). 